\title{
Ethical Guidelines for Artificial Intelligence in Healthcare from the Sustainable Development Perspective
}

\author{
Anrī Leimanis ${ }^{1}$ and Karina Palkova ${ }^{2}$
}

\begin{abstract}
Use of Artificial Intelligence (AI) in variety of areas has encouraged an extensive global discourse on the underlying ethical principles and values. With the rapid AI development process and its near instant global coverage, the issues of applicable ethical principles and guidelines have become vital. AI promises to deliver a lot of advantages to economic, social and educational fields. Since AI is also increasingly applied in healthcare and medical education areas, ethical application issues are growing ever more important. Ethical and social issues raised by AI in healthcare overlap with those raised by personal data use, function automation, reliance on assistive medical technologies and the so-called 'telehealth'. Without well-grounded ethical guidelines or even regulatory framework in respect of the $\mathrm{AI}$ in healthcare several legal and ethical problems at the implementational level can arise. In order to facilitate further discussion about the ethical principles and responsibilities of educational system in healthcare using AI and to potentially arrive at a consensus concerning safe and desirable uses of AI in healthcare education, this paper performs an evaluation of the self-imposed AI ethical guidelines identifying the common principles and approaches as well as drawbacks limiting the practical and legal application of internal policies. The main aim of the research is to encourage integration of theoretical studies and policy studies on sustainability issues in correlation between healthcare and technologies, the AI ethical perspective.
\end{abstract}

Keywords: artificial intelligence, healthcare, ethical AI, guidelines, sustainability.

\section{Introduction}

AI's core application is to autonomously perform tasks that traditionally require human intelligence. According to many prominent thinkers of our time, AI will deeply impact the society in a number of aspects sooner rather than later. The World Economic Forum in 2019 concluded that "as rapid advances in machine learning increase the scope and scale of AI's deployment across all aspects of daily life, and as the technology can learn and change on its own, multi-stakeholder collaboration is required to optimize accountability, transparency, privacy and impartiality to create trust." (AI report, 2020). AI's tools that can be used in the healthcare sector grow extremely fast. Electronic medical records of patients are used for machine learning. Exponential growth of available medical data allows AI algorithms to rapidly improve in precision to detect various anomalies and over time become increasingly more accurately than human medical practitioners, especially in the field of cardiology and oncology. It can be concluded that technological impact, including AI, has set course for significant transformations in the healthcare system and creating a new technological era in medicine. Integration of technology into 
the traditional healthcare system laid down challenges from the both technological and ethical aspects (Chi -Tung Cheng, 2020).

While AI has proven to produce a lot of advantages there are certain risks and challenges arising out of AI use in healthcare that can be divided into three groups.

The first are legal or regulatory issues such as registration, certification of technology and medical and non-medical staff and, of course, legal liability.

The second group of issues are technological but related to different set of rules for use of private medical data in various jurisdictions i.e. limited use of personal medical data potentially may lead to race, gender and age technological biases that in turn may mean inadequate results for various social groups and ineffectiveness of medical treatments. Third group are ethical issues.

Both ethics and law from the AI sustainable development perspective play vital role in the socio-economic situation in the world. If legal problematic on AI level is more material and connected to international and national policies and regulatory frameworks with the aim to ensure that AI based technologies can benefit society as a whole, than Ethical issues are common on national and international level and cannot be brought under the legal control from the national or international legal perspective. AI ethical issues in healthcare are complicated from procedural perspective. From the one hand general ethical principles with regard to new technological approach in healthcare allows us to better understand the overlaps and differences between modern technologies, socio economical responsibility and $\mathrm{AI}$ ethics, national and international government regulation. From the other hand the are challenges from the responsibility and decision-making perspective comes out (Saveliev \& Zhurenkov, 2020). Ethical AI issues are inextricably linked to modern society especially in healthcare system, where ethical issues are historically important 9DeepMind. DeepMind Ethics). The duties and qualifications of medical practitioners were never more fully exemplified than by the conduct of Hippocrates, or more eloquently described than by his pen. Medical Ethics of Hippocrates shows the importance of particular institute (Brody, Meghani \& Greenwald, 2009). In shall be noted, that many legal decisions in past as well as in nowadays follow from the perception of certain ethical problems. And if before the society were able to speak about the human ethics which have been studied, that AI ethics in healthcare is one of latest studies, that still in progress. AI gives a new direction to these ethical questions. Systems that are used in healthcare become more autonomous and the potential impact on the human health is not researched enough. The AI ethics is the subject of sustainable development on healthcare level. But the challenge is to understand the logical or systematic AI's based technologies reaction and functioning. From theoretical perspective the main task of AI shall correlate with the human-centred positive needs. The human beings shall be protected from the non-ethical AI based technologies behaviour AI. AI must act in the best interests of the society. To achieve this goal global standards or ethical setting instruments can be provided. This standard must promote human rights, dignity and transform understand of ethical principles to uninfected guidelines centered to health care based sustainable development.

In the future AI and health care will be as an indivisible mechanism. As mentioned above medical practitioners, medical students as well as patients will need some extra or unique knowledge in current medical practice. From the patients - health care practitioners' 
perspective AI provided medical information and data issues will be discussed during the health care process. But this information cannot be viewed separately from ethical issues. Medical practitioners can be supervisors of the AI provided information. AI can not provide several services to patients yet, for instance in the case of emotional support whet the patients shall be informed on diagnosis. AI do not "feel "the situation. There are algorithms that coordinate the behaviour. It means, that medical practitioners need to acquire communication knowledge, other skill connected to emotional side.

Advances in AI applications to education have prompted widely covered global discourse on the underlying ethical principles and values. In the absence of national and international regulatory framework for AI, numerous academic and industrial research institutions, standards-setting bodies, professional bodies and non-governmental entities around the globe have published their own guidelines and policies for ethical deployment of AI (White Paper On Artificial Intelligence, 2020).Even though the aim for most of the guidelines is to maximize the benefits that AI delivers, the policies differ significantly in content and application (Beijing Academy of Artificial Intelligence, 2019).

The European Union's white paper on AI acknowledges a number of potential risks related to AI's use, such as opaque decision-making, gender-based or other kinds of discrimination, intrusion in private lives or being used for criminal purposes. The white paper is only a set of guidelines, the European Commission is expected to start drafting binding legislation after proper feedback analysis [Brussels, 19.02.2020] According to the EC, trustworthy AI should be lawful, ethical and robust i.e. (Ethics Guidelines for Trustworthy AI, 2019). AI shall respect all applicable laws and regulations, ethical principles and values, and consider its social environment. The EC Guidelines list seven key requirements that AI systems should meet in order to be deemed trustworthy: human agency and oversight, technical robustness and safety, privacy and data governance, transparency (the data, system and AI business models should be transparent), diversity, non-discrimination and fairness, societal and environmental well-being (AI systems should benefit all human beings, including future generations) and accountability (to ensure accountability for AI systems and their outcomes) (Information Technology Industry Council, 2017).

International organizations, including the Council of Europe, the United Nations Educational Scientific and Cultural Organization (UNESCO), the Organisation for Economic Co-operation and Developments (OECD), the World Trade Organisation and the International Telecommunications Union (ITU), UN's High-Level Panel on Digital Cooperation and others are busy discussing and producing their own ethical AI guidelines and principles (Microsoft Corporation, (2019).

An increasing number of academic and commercial entities - developers and deployers of AI - have embraced on a journey of setting self-imposed ethical guidelines for AI thus acknowledging the importance of designing AI with human rights, consumer protection, and product safety and liability rules in mind. Amongst the most influential and far reaching guidelines are the so-called ASILOMAR AI Principles, Beijing AI Principles, Google's (Google, 2018). Perspectives on Issues in AI Governance, Microsoft AI Principles, OpenAI Charter, and Partnership on AI. (Asilomar Conference 2017).

Such privately debated and approved ethical guidelines play an important role in AI design and application in healthcare (Google, 2019). The guidelines provide certain framework 
for researchers and education providers to anticipate the possible ethical issues (Organisation for Economic, 2019). Importantly the guidelines in majority of cases stipulate that ethical issues must be evaluated when AI-based educational activities are planned (Ethics for AI, 2020).

It must be observed that the sheer number of academic, commercial, and governmental bodies working on ethical AI principles in healthcare makes it difficult to track the actual impact on decisions of AI developers and AI-enabled tools produced. While there is a complete lack of reinforcement mechanisms, deviations from the self-imposed guidelines are difficult to identify (OpenAI, 2018). The lack of empirical evaluation of AI tools' implementation societal effects makes it difficult to measure effectiveness of such internal policies, not to mention the risks related to commercial rewards - it cannot be excluded that ethical guidelines may serve for marketing purposes (Partnership on AI, 2018).

\section{Methodology}

For the purposes of research is to identify the main theoretical problematics of ethical guidelines for artificial intelligence in healthcare from the sustainable development perspective.

The methods of analysis and induction are used. The authors will provide the legal analysis considering international regulations. In order to complete, the research the authors are using general scientific methods such as synthesis, modelling, comparative method and deductive method. Theoretical base of the study is constituted by contemporary international scientific works and the articles of national and international authors. As well as some authors, scientists who have provided global contribution to the development of Ethics AI will be provide. The object of the research is to encourage integration of theoretical studies and policy studies on sustainability issues in correlation between healthcare and technologies from the AI ethics perspective.

\section{Research.}

\subsection{Applications of AI in healthcare Education}

The definition of AI in correlation with the healthcare was used in 1984. AI in healthcare was defined as a particular mechanism of several AI based programs that can diagnose and as well as recommend to patients or doctors certain types of therapy (Coiera, 1998).

Development of technology particularly the machine learning has created many new opportunities in the field of education, particularly healthcare education. Medical students are not so distant future will benefit from AI-powered mixed reality and computer vision solutions that can provide an immersive environment to stimulate interest and understanding, while the simulations will encourage student engagement and enhance learning in more intuitive and adaptive ways. As in any other area of study medical students will benefit from technology instantly connecting professors across the globe to their classrooms or scientific discovery laboratories. University administrative processes will benefit from utilizing AI on the large amounts of data produced during research and 
teaching as well as student and young medical practitioners' performance monitoring tools that will allow more responsive teaching planning and individualization of programs.

A number of universities across the globe have already started implementation of AIenabled tools to their research and teaching processes. Technical University of Berlin in Germany develops AI-powered chat-bots using natural language applications to take over routine academic tasks such as grading assignments, answering questions from students and so forth. Carnegie Mellon University in the US develops AI-based cognitive tutors for the statistics course with an aim to minimize student - instructor contact, thus achieving comparable learning results with higher effectiveness of resources employed. Georgia State University in the US tracks individual student performance to predict evaluations and the need for interventions to allow students to reach their full potential and prevent students from dropping out. University of Aberystwyth in Wales employs an AI technology that performs the scientific process based on its own judgement - from formulation of hypotheses, to design of experiments, performance of experiments, data analysis and finishes with a decision on further research strategy (Chi -Tung Cheng, 2020).

It shall be mentioned that important role in AI and education from the healthcare development perspective plays training programs for medical practitioners. There are several key values for training prospective medical practitioners as science, technologies. Medical education is on the transformation process. There are new central aspects become into the game. Medical practitioners must consider innovations, robotics, AI based healthcare equipment etc. For that reason, theoretical and practical knowledge of AI based technologies shall be part of educational system.

Fast growing innovation in AI affects medical practice, as well affects training mechanism implemented in the future medical practitioners' sustainable development. As AI technologies in health care system develop very fast the key issue is what kind of specific technical knowledge medical students shall know. From that perspective the importance of new type of knowledge for medical students starts from technical use and understanding of AI based technologies and ends with the data protection issues or data security issues. The authors agree with the Liam G. McCoy and co-authors, who argue that medical practitioners need to understand AI logic and basic technology specific that impacting clinical decision-making. The are several important skills and professional information shall be provided during the medical students' educational process. Medical students, or medical professionals during the educational process shall understand and identify the situation when the technology is the better way for a given clinical context. The issue of interpretation and understanding of the information received by AI based technologies is another important issue.

The results given by the AI in particular health care-based situations shall be analized with the accuracy. The information on medical error, clinical inapplicability shall be considered. Medical practitioners, medical students shall be protected and educated enough to be able to explain the results received by AI based systems. So, patients and medical practitioners considering the good practice of communication and informed consent between too parties shall be ready for communication and discussion. If medical practitioners used AI provided information, he must have the skills to clarify and explain all necessary technical as well medical information provided by AI to patient (McCoy, Nagaraj, Morgado, F. et al., 2020). 
Based on mentioned before medical practitioner can detect several problems the technical as well ethical point of view. AI can be characterised by the term of technical or ethical non explainability.

Therefore, to ensure the best interests of patients and provide correct and ethical, professional services during fast growing technological progress in health care services educational system shall be improved. AI based technologies and rights, ethical issues in short period of time will take one of the central roles in the future health care system, where AI will have bigger power as data provider to medical practitioners and as developer of AI driven big data processing (Law, Veinot, Campbell, Craig \& Mylopoulos, 2019).

As practice shows knowledge based on innovations in collaboration with the medical practitioners 'practical knowledge can give better result as for science in research field, as well for society. (Prober \& Khan, 2013). There is one aim for health care professionals -to take care on patients. AI can help to achieve the aim but for medical practitioner, medical students important is to acquire skills based on technical requirements. Therefore, special technological medical schools, medical education technology centres can be created to make the level of health care education more compliance to nowadays situations.

It must be mentioned, that in common, the question on AI in health care and patients' rights is not solved from the ethical and legal point of view as well. If AI legal aspects can be clarified and issue on liability solved by national level according to national regulations, that ethical aspect is an international problem and challenge. As documents snow the standards that laid down information on AI use in health care are still in progress. But on educational level this standard can be simulated and viewed in the context of existing clinical care standards, quality standards, malpractice etc. More AI based technologies come into the relations between medical practitioners and patients. Patients actively use chatbots, health care applications, etc. Medical practitioners can use that tools as well. But the issue on ethical standards and AI ethics in common comes out (Paranjape, Schinkel, Nannan Panday, Car\& Nanayakkara, 2019).

The current question is how existing educational system and task can be transformed and updated to provide more effective approach to AI issue in healthcare. New realities show new challenges, and the central role of the challenges play AI ethical issues. Medical education nowadays provides more frameworks that shall be updated.

According to High-Level Expert Group on AI Ethics Guidelines there are several key points. The Expert group provide following vital AI ethical principles. AI shall be lawful, it means that AI shall respect all applicable national and international laws and regulations, legal documents. AI shall be ethical, and it means that AI shall respect ethical principles and values from the different educational area perspective as well. If AI is applicable at medical education or medical training level, the principles of medical ethics must be considering and respected. (Ethics guidelines for trustworthy AI, 2019).

Important issue from the medical education perspective is the use of AI systems in health care processes. This process must be secured. Patient's data is vital and sensitive information has to be protected on the highest level. Ensuring total respect for patient's data protection and the mechanisms the quality and integrity of the data must be taking into account. Therefore, the AI ethical guidelines for patient's privacy and data protection at educational level shall be provided (Ethics guidelines for trustworthy AI, 2019). Important AI ethical principle from the medical education perspective is connected to 
Transparency. This principle in healthcare situation shows that the data and AI systems used in healthcare should be transparent. The patients must know and understand the process of AI provided decisions. Moreover, this must be explained to the patients in good and understandable manner to whom this concerned. For that reason, medical practitioners shall know the concept of AI system, as well about the limitations of this system. In common, all AI ethical principles provided by European Commission's HighLevel Expert Group can be implemented in healthcare level. The spatial, more detailed interpretation of particular principles is needed. In common AI ethical guidelines provided by scientists, European Commission etc., meet the Medical Ethics requirements or standards. Nevertheless, technical improvements and special explanation shall be provided on national and international level to unify existing policy planning documents.

\subsection{Global discourse on ethical AI from the Sustainable Development perspective and liability issues}

On April 25, 2018, the European Commission has adopted a communication "Artificial Intelligence for Europe" which referred to the need not only to assess the impact of new digital technologies, including AI, on the current liability regime, but also to identify and explore possible gaps in the AI liability regime and its potential consequences (Commission Staff working document, 2019). The issue of the AI liability is based on the AI safety considerations, which is also highlighted in the European Commission's Communication "Building Trust in Human-Centric Artificial Intelligence" (Building Trust in Human-Centric Artificial Intelligence, 2019).

The Communication states that AI systems must have integrated safety and security-bydesign mechanisms. Based on the recommendations of the experts of the European Union and the European Union policy in general, the responsibility for the AI system as an object should lie with the owner of the object. If AI technology, identifying specific gene mutations from images of tumour pathology rather than traditional genome sequencing, makes a mistake, the issue of liability would be considered primarily taking into account the nature of the technology's technical error and the owner of the technology. However, it should be noted that the owner of AI technology can obtain it from the manufacturer, who will not always be competent in the technical nuances of AI. Changes to AI technology can also be made by service companies. The services provided may affect the operation and results of the machine. Therefore, in case AI has caused harm to the patient by its decision, there is a debatable question regarding the liability, whether it will be assumed by the manufacturer, service provider, owner or user (Neri, E., Coppola, F., Miele, V. et al., 2020). If the issue of AI liability in the context of treatment is viewed from the perspective of, for instance, product liability, then natural and legal persons have the right to compensation upon the occurrence of the specified conditions (discussed above). In fact, regulation is able to minimize the risk of harm to users and is intended to ensure compensation for harm caused by, for example, defective goods.

Despite the existing liability regime and policy direction in general, there are a number of challenges regarding the matter of liability in the context of AI medical treatment. Firstly, the application of AI in medical treatment is a complicated process from both a technological and practical point of view, as well as from an ethical point of view. 
The Committee on Legal Affairs of the Council of Europe, in its report on Civil Law Rules on Robotics, in paragraph 59 (f) has recommended to consider the possibility of "creating a specific legal status for robots", which could also address ethical issues in healthcare. This also applies to AI robotic technologies used in healthcare. The report states that the most complex cognitive robots could obtain a new legal status with certain responsibilities, rights, and obligations. Given the rapid development of AI, especially in the field of healthcare, it is clear that scientists, philosophers, futurists and lawyers will return to the question of the legal status of AI in the near future, specifically in the context of liability and ethics. The legal issues of AI are closely related to ethics.

AI ethics is currently associated with various types of "concerns". It is a predictable and explainable reaction to new technologies. Issues of concern to scientists, lawyers, doctors, other specialists and the society as a whole are most often ranging from AI's ability to communicate kindly to confidence in non-disclosure of information to third parties.

The use of AI in healthcare is forming a new approach that could relieve pressure on healthcare professionals or potentially create competition. Despite the large number of studies and the development potential in the field of healthcare, ethical issues have raised new management requirements. The most important aspects are the strengthening of ethical principles and the liability of interested parties in the ethical governance system.

AI can already accurately diagnose skin cancer and compete with a certified dermatologist (Esteva, Kuprel, 2017). AI can do it faster and more efficiently because it requires a set of training data that includes years of experience and case analysis of health professionals. AI can be used in almost any field of medicine and has the potential to contribute to biomedical research, medical education, and healthcare delivery.

In order to qualify a problem as an AI ethical problem, it is necessary to identify which actions are considered "right" and "wrong", which are "ethical actions" and "unethical actions", as well as to understand the concept of ethics in a medical context.

In general, ethics is understood as a doctrine of morality, that is, careful and systematic reflection on decisions and behaviour of a moral nature, as well as their analysis in the past, present, and future (World Health Communication Associates, 2009). If ethics is considered in the context of medicine, it means the field of ethics, which deals with issues of a moral nature in medical practice, including in medical treatment.

In turn, the explanation of IA ethics can be found in the European Commission's Ethics Guidelines for Trustworthy AI, which state that AI ethics is a sub-field of business ethics that addresses the ethical issues raised by the development, deployment and use of AI. Its main objective is to identify whether AI can improve living standards of individuals or raise concerns about them in terms of quality of life or the necessary independence and freedom of people in a democratic society (Guidelines for Trustworthy AI, 2019).

It can be concluded from the above that, in essence, both the understanding of ethics in medicine and the ethics of AI are aimed at solving similar issues yet using different approaches to achieve the goals. The ethical problem, as well as the ethical problem of AI, must be based on a precondition, which indicates that an action taken in specific circumstances is not morally permissible. An important aspect of AI ethics is societal values, moral and ethical considerations, which help to determine the specific value priorities of different interested parties in different multicultural contexts, to explain the rationale for the decision and guarantee its transparency. However, with regard to morality, 
there is a question of whether it is possible to grant moral status to AI, especially in medical treatment. Today, AI acts more as a performer of moral action, yet will AI be able to experience the moral dignity and, for example, protection against harm that patients currently experience. It is a sound argument that most people are both performers of moral conduct and subjects of moral interest. But there are exceptions. For instance, infant patients or patients in a coma are only subjects of moral interest.

When AI technologies capable of making decisions based on ethical behaviour will be developed, the challenge will be whether AI can fall into the category of moral performers, which will also affect the issue of AI liability.

Given the complexity of AI, one of the most important issues, especially from an ethical point of view, is to guarantee safety and protection to a patient - the fundamental principle underlying human rights, so it is needed to trace or to explore the logic of all actions taken by AI, or the reasons and causes for non-action. It is also important in the context of AI's ethical liability.

A significant step in the context of ethical issues is the European Parliament's report with the recommendations to the Commission on the Civil Law Rules on Robotics (Civil Law Rules on Robotics, 2017). The document referred focuses more on ethics of robotics, but similar issues are identified in the field of AI ethics. Specifically, the European Commission's AI working group's Ethics Guidelines for Trustworthy AI with the primary aim of promoting trustworthy AI should be highlighted in the matters of AI ethics. At the same time, three necessary components shall be noted, namely:

1. AI must be legal and comply with all applicable laws and regulations.

2. $\quad$ AI must be ethical, i.e., respect of ethical principles and values must be ensured.

3. AI must be technically and socially sustainable, as AI systems can cause unintentional harm, even if the intentions have been good. (A definition of AI, 2019).

The guidelines also set out the main requirements to be met in order for AI to act ethically and credibly, and these are: Human agency and oversight, Technical robustness and safety, Privacy and data governance, Transparency, Diversity, non-discrimination and fairness, Environmental and societal well-being, Accountability (A definition of AI, 2019).

These requirements are necessary in order to ensure the ethical principles of AI, which are vital in the medical treatment process in the context of the patients' rights protection. There are several views on the classification of ethical principles in AI, but the generally accepted ones are:

1) the principle of dignity,

2) the principle of privacy,

3) the principle of autonomy,

4) the principle of responsibility,

5) the principle of non-harm,

6) the principle of doing good,

7) the principle of justice.

The ethical principles of AI in question are not exhaustive but comparing them with the generally accepted principles of medical ethics, a similarity can be concluded.

Four basic principles dominate in medical ethics: the principle of personal autonomy, the principle of non-harm, the principle of doing good and the principle of justice. These principles are closely interlinked and complementary. The ethical principles of AI must 
also be considered in context, considering their complementary nature. The ethical principles of AI and medical professionals are based on identical elements, which by their similarity ensure the protection of the patient's rights and interests also in the context of ethics. Today, AI is not autonomous and operates under the supervision of medical professionals. It should be noted, however, that the ethics of AI in medical treatment is a relatively new direction, and it is possible that the content of AI ethics will be transformed. The incompleteness of the AI ethical issues, that are intricately connected to AI liability show that AI cannot not replace the medical practitioners yet. From that point of view in the near future medical practitioners will be the only who will take all key decisions in healthcare.

But AI, as important digital assistants will increasingly enter medical practitioners' professional lives. The task of the AI is to minimize the probability of medical error. The whole point of introducing AI into medicine is to help the medical practitioner and save the professionals from routine. Nevertheless, the AI in healthcare can be divided or understanded from the two perspectives. The first one is the AI as assistants for patients and the second one the AI for medical practitioners. If the patients use AI for treatment procedures, for instance "Dr. Google" ( Kłak, Gawińska, Samoliński, Raciborski, 2017), than this is a zone of "responsible self-treatment". The patients are responsible for the results, they agreed on decisions made by AI. But the AI for doctors means that the AI systems usually are as "medical decision support systems", but not decision makers.

\section{Conclusion and Implications}

The future development of AI ethics in healthcare specifically is a difficult and very important question from the perspective of society's well-being.

Considering how conveniently and affordably certain AI technology applications to healthcare have become, it is evident that patients will become more involved in healthcare processes themselves. It can be predicted that medical practitioners will play the role of providers in the treatment process and will collaborate with the technology providers and patients in gathering and processing medical data. Medical practitioners will have to be able to explain the treatment decisions that will be based on AI recommendations. It will in turn require significantly higher level of understanding by the medical staff of the underlying technology, including AI applications, both from technological, medical and regulatory aspects. Hence an important role in sustainable and ethical development of technology in healthcare will be played by the education system. Medical students will not only study the technology's application to healthcare services, but medical education will use AI to enhance the study process.

Implementation of AI solutions in a host of areas from industry to medicine, from justice system to education has encouraged an extensive global discourse on the underlying ethical principles and values. With the rapid AI development process and its near instant global coverage and deployment, the issues of ethical principles underlying the algorithms has attracted the public's eye as vital.

AI delivers significant increase in productivity in the healthcare with the rise of advanced solutions for medical data use for machine learning, healthcare function automation, 
assistive medical technologies and more precise and remote medical diagnosis tools enabling prevention of diseases.

Technology developers in many cases are not bound by the regulatory framework applicable to institutional healthcare providers and their personnel as well as healthcare education providers. In order to narrow the regulatory gap and limit the risks, many of the AI developers have adopted internal self-imposed AI ethics guidelines and policies. But such approach has very clear drawbacks. Patients most certainly will experience difficulty accessing and understanding the mix of applicable government regulations with the private self-imposed ethical principles of a myriad of players involved in provision of modern technologically advanced healthcare service such as: (i) developer of technology that collects the medical samples and transforms results into data for machine learning, (ii) AI application developer, (iii) system operator at the medical centre, and finally (iv) medical practitioner administering drugs or treatment based on AI recommendations.

In order to facilitate further discussion about the ethical principles, responsibilities of educational institutions using AI, and to potentially arrive at a consensus concerning safe and desirable uses of AI in education, an evaluation of the current and upcoming AI ethical guidelines is desirable.

Without well-grounded ethical guidelines or even regulatory framework in respect of the AI in healthcare several legal and ethical problems at the implementational level can arise. In order to facilitate further discussion about the ethical principles and responsibilities of educational system in healthcare using AI and to potentially arrive at a consensus concerning safe and desirable uses of AI in healthcare education, an evaluation of effects of self-imposed AI ethics guidelines identifying the common principles and approaches as well as drawbacks limiting the practical and legal application of such policies.

In order to guarantee implementation of ethical AI in medical education and in healthcare in common the providers will have to figure out how can humans most effectively perform research and study side by side with systems built by humans but constantly upgraded by systems themselves. It is likely that AI-enabled technology will take an ever-greater place in medical education, hence the need of regular evaluation of ethical AI guidelines and internal policies, matched by sufficient governmental regulatory intervention in case selfimposed guidelines will prove insufficient to guarantee human basic rights. And finally, AI ethics shall be closely connected to the dominate principles in medical ethics. The AI is not ready to be the main decision maker in healthcare, but the AI can be the good digital assistants for medical practitioners.

\section{References}

Artificial intelligence: improving man with machine. 2020. Retrieved from https://reports.weforum.org/digital-transformation/artificial-intelligence-improving-man-withmachine/ Accessed 12.12.2020.

Building Trust in Human-Centric Artificial Intelligence, 2019. COMMUNICATION FROM THE COMMISSION TO THE EUROPEAN PARLIAMENT, THE COUNCIL, THE EUROPEAN ECONOMIC AND SOCIAL COMMITTEE AND THE COMMITTEE OF THE REGIONS. Retrieved from: https://eur-lex.europa.eu/legalcontent/EN/TXT/?uri=CELEX\%3A52019DC0168 Accessed 12.12.2020.

COMMISSION STAFF WORKING DOCUMENT Liability for emerging digital technologies Accompanying the document Communication from the Commission to the European Parliament, 
the European Council, the Council, the European Economic and Social Committee and the Committee of the Regions Artificial intelligence for Europe. Retrieved from: https://eurlex.europa.eu/legal-content/EN/TXT/PDF/?uri=CELEX:52018SC0137\&from=en Accessed 12.12.2020.

Chi -Tung Cheng, et al. Artificial intelligence-based education assists medical students' interpretation of hip fracture. INSIGHTS INTO IMAGING Volume: 11 Issue: 1 Article Number: 119 Published: DEC 232020 Retrieved from https://insightsimaging.springeropen.com/track/pdf/10.1186/s13244-020-00932-0.pdf Accessed 12.12.2020.

Coiera., W.,E. Artificial Intelligence in Medicine: The Challenges Ahead // Journal of the American Medical Informatics Association, 1996, Vol. 3, N 6., p. 363 - 366.

Saveliev, A, Zhurenkov, D Artificial intelligence and social responsibility: the case of the artificial intelligence strategies in the United States, Russia, and China. KYBERNETES, DOI: 10.1108/K-01-2020-0060, DEC 2020. Retrieved from https://www.emerald.com/insight/content/doi/10.1108/K-01-20200060/full/html. Accessed 12.12.2020.

Brody H., Meghani Z., Greenwald K., Michael Ryan's Writings on Medical Ethics. Michael Ryan's Writings on Medical Ethics. Medical Ethics of Hippocrates Springer, Dordrecht, 2009. 117-128 p.

European Commission. REPORT FROM THE COMMISSION TO THE EUROPEAN PARLIAMENT, THE COUNCIL AND THE EUROPEAN ECONOMIC AND SOCIAL COMMITTEE Report on the safety and liability implications of Artificial Intelligence, the Internet of Things and robotics. Brussels, 19.02.2020. Retrieved from https://ec.europa.eu/info/sites/info/files/report-safetyliability-artificial-intelligence-feb2020_en_1.pdf. Accessed 12.12.2020.

European Commission. High-Level Expert Group on AI presented Ethics Guidelines for Trustworthy Artificial Intelligence (2019). Retrieved from: https://ec.europa.eu/digital-singlemarket/en/news/ethics-guidelines-trustworthy-ai Accessed 21.12.2020.

European Parliament. Report with recommendations to the Commission on Civil Law Rules on Robotics (2015/2103(INL)). Retrieved from: https://www.europarl.europa.eu/doceo/document/A-82017-0005_LV.html Accessed 21.12.2020.

Esteva, A., Kuprel, B. (2017). Dermatologist-level classification of skin cancer with deep neural networks. Nature. 115-118. 542(7639).

"American AI Initiative" (US President's Executive Order, 2019). Retrieved from https://www.whitehouse.gov/ai/ Accessed 21.12.2020.

ASILOMAR AI Principles (Asilomar Conference 2017). Retrieved from https://futureoflife.org/aiprinciples/. Accessed 21.12.2020.

Ethics for AI. University of Oxford. (2020) Retrieved from: https://www.cs.ox.ac.uk/efai/ Accessed 21.12.2020.

Beijing Academy of Artificial Intelligence (2019). Beijing AI Principles. Retrieved from https://www.baai.ac.cn/blog/beijing-ai-principles Accessed 21.12.2020.

DeepMind. DeepMind Ethics \& Society Principles. 2018. Retrieved from https://deepmind.com/applied/deepmind-ethics-society/principles/ Accessed 21.12.2020.

Google. (2018). Artificial Intelligence at Google: Our Principles. Retrieved from https://ai.google/principles/ Accessed 21.12.2020.

Google. (2019). Perspectives on Issues in AI Governance. Retrieved from https://ai.google/static/documents/perspectives- on-issues-in-ai-governance.pdf: 1-34

High-Level Expert Group on Artificial Intelligence. A definition of AI: Main capabilities and scientific disciplines. (2019). Brussels. p. 6. Retrieved from https://ec.europa.eu/digital-singlemarket/en/news/definition-artificial-intelligence-main-capabilities-and-scientific-disciplines Accessed 21.12.2020.

Information Technology Industry Council. (2017). ITI AI Policy Principles. Retrieved from https://www.itic.org/public- policy/ITIAIPolicyPrinciplesFINAL.pdf Accessed 21.12.2020.

Anna Kłak, Emilia Gawińska, Bolesław Samoliński, Filip Raciborski. Dr Google as the source of health information - the results of pilot qualitative study, Polish Annals of Medicine, (2017), Volume 24, Issue 2, Pages 188-193, ISSN 1230-8013, https://doi.org/10.1016/j.poamed.2017.02.002.

Law, M., Veinot, P., Campbell, J., Craig, M. \& Mylopoulos, M. Computing for medicine: can we prepare medical students for the future? Acad. Med. 94, 353 (2019). 
Microsoft Corporation. (2019). "Microsoft AI Principles." Retrieved from https://www.microsoft.com/enus/AI/our-approach-to-ai Accessed 21.12.2020.

McCoy, L.G., Nagaraj, S., Morgado, F. et al. What do medical students actually need to know about artificial intelligence?. npj Digit. Med. 3, 86 (2020). https://doi.org/10.1038/s41746-020-0294-7

Neri, E., Coppola, F., Miele, V. et al. Artificial intelligence: Who is responsible for the diagnosis?. Radiol med 125, 517-521 (2020). https://doi.org/10.1007/s11547-020-01135-9

OpenAI. (2018). OpenAI Charter. Retrieved from https://openai.com/charter/ Accessed 21.12.2020.

Partnership on AI. (2018). About Us. Accessed https://www.partnershiponai.org/about/ Accessed 21.12.2020.

Paranjape, K., Schinkel, M., Nannan Panday, R., Car, J., \& Nanayakkara, P. (2019). Introducing Artificial Intelligence Training in Medical Education. JMIR medical education, 5(2), e16048. https://doi.org/10.2196/16048

Prober, C. G. \& Khan, S. Medical education reimagined: a call to action. Acad. Med. 88, 1407-1410 (2013).

The European Commission's High-Level Expert Group on Artificial Intelligence: Ethics Guidelines for Trustworthy AI. 2019. Accessed https://ec.europa.eu/digital-single-market/en/news/ethicsguidelines-trustworthy-ai Accessed 21.12.2020.

The European Commission's White Paper On Artificial Intelligence - A European approach to excellence and trust. Accessed https://ec.europa.eu/info/sites/info/files/commission-white-paper-artificialintelligence-feb2020_en.pdf Accessed 21.12.2020.

Organisation for Economic Co-operation and Development. (2019). Recommendation of the Council on Artificial Intelligence. Accessed https://legalinstruments.oecd.org/en/instruments/OECDLEGAL-0449 Accessed 21.12.2020.

Palkova., K. Robottehnologíiju ietekme uz veselïbas aprūpes sistēmu. (2019). SOCRATES. Rīgas Stradiņa universitātes Juridiskās fakultātes elektroniskais juridisko zinātnisko rakstu žurnāls / Rīga Stradinsš University Faculty of Law Electronic Scientific Journal of Law Socrates. 2019, 2 (14). https://doi.org/10.25143/socr.14.2019.2.047-052.

Retrieved from https://dspace.rsu.lv/jspui/bitstream/123456789/937/1/IPD-84-211_Socrates-142019_04-Palkova_047-052.pdf Accessed 21.12.2020.

World Health Communication Associates. Medical ethics. (2009). Retrieved from http://www.medicinasapgads.lv/userfiles/files/Medicinas\%20etika.pdf Accessed 21.12.2020 